\title{
Response to Comment on Effective Temperature and Structural Rearrangement in Trapped Ion Mobility Spectrometry
}

Denis Morsa, ${ }^{1}$ Emeline Hanozin, ${ }^{1}$ Valérie Gabelica, ${ }^{2}$ and Edwin De Pauw ${ }^{1 *}$

${ }^{1}$ Mass Spectrometry Laboratory, MolSys Research Unit, University of Liège, Liège, 4000, Belgium ${ }^{2}$ Univ. Bordeaux, CNRS, INSERM, ARNA, UMR 5320, U1212, IECB, F-33600 Bordeaux, France.

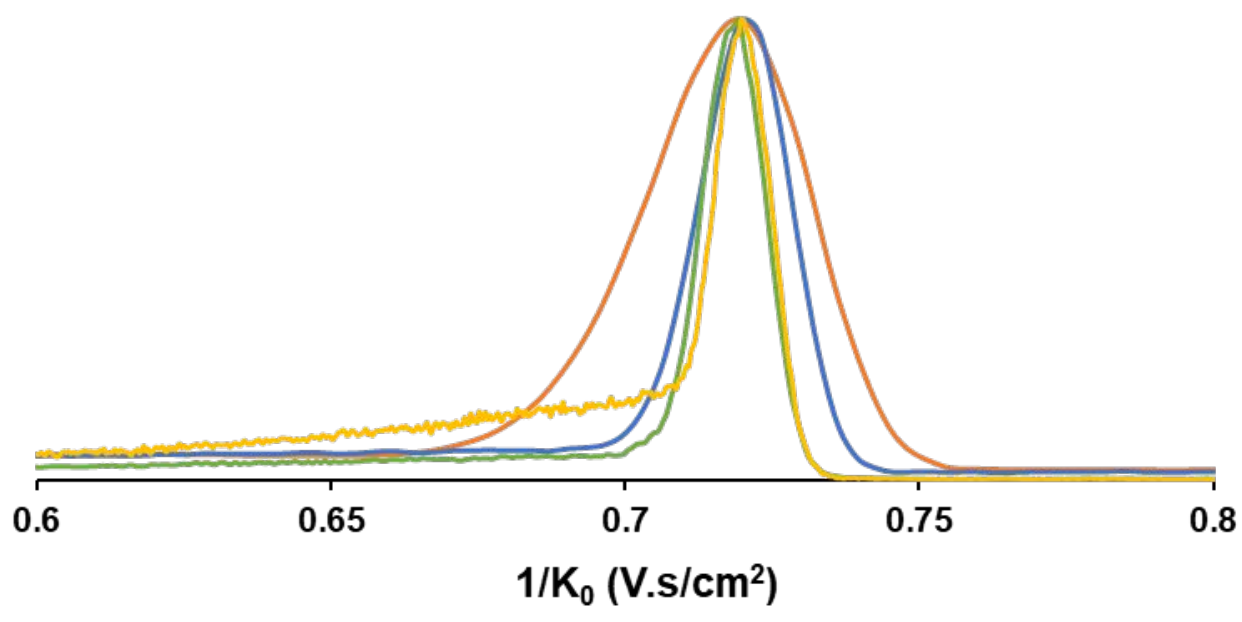

$-12 \mathrm{~ms}-25 \mathrm{~ms}-100 \mathrm{~ms}=200 \mathrm{~ms}$

Figure S1. Extracted ion mobility spectra (EIM) of $\mathbf{M}^{+}$monitored on $\mathbf{p}-\mathbf{O M e}$ as a function of the analysis time with $100 \%$ duty cycle on the timsTOF Pro. The peak width narrows with increasing time and tailing is not observed at $100 \mathrm{~ms}$. 